\title{
Psychische Symptome bei Studentinnen in Ost- und Westdeutschland: Eine Replikation nach 10 Jahren
}

\author{
A. Dinkel ${ }^{a}$ \\ H. Berth ${ }^{a}$ \\ C. Exner ${ }^{b}$ W. Rief ${ }^{b}$ \\ F. Balck ${ }^{\mathrm{a}}$ \\ a Universitätsklinikum Dresden, Medizinische Psychologie \\ b Philipps-Universität Marburg, Klinische Psychologie und Psychotherapie, Deutschland
}

\section{Schlüsselwörter}

Ost-West-Vergleich · Psychische Symptome

Befindlichkeit · Studenten

\section{Zusammenfassung:}

Hintergrund: Nach der deutschen Wiedervereinigung beschäftigte sich eine Reihe von Studien mit der psychischen Befindlichkeit der Menschen in Ost- und Westdeutschland. Die vorliegende Untersuchung stellt eine Replikation der Studie von Basten et al. [Psychische Beschwerden und körperliche Gesundheit im vereinten Deutschland: Eine OstWest-Vergleichsuntersuchung an einer studentischen Stichprobe. Verhaltenstherapie 1994;4:90-95] 10 Jahre nach der Originaluntersuchung dar. Diese wurde 1991/1992 durchgeführt und untersuchte Unterschiede in der Depressivität, Angst, Somatisierung sowie im berichteten Essverhalten zwischen Studentinnen in Dresden und Marburg. Probanden und Methode: Von Oktober 2001 bis April 2002 wurden die psychischen Beschwerden und das Essverhalten bei Dresdner und Marburger Studenten untersucht. In die vorliegende Arbeit gingen Daten von 221 Studentinnen ein. Es wurden dieselben standardisierten Erhebungsinstrumente wie in der Originaluntersuchung eingesetzt. Ergebnisse: Im Unterschied zu der Originalstudie konnte keine höhere Depressivität bei der westdeutschen Stichprobe nachgewiesen werden. Stattdessen gaben ostdeutsche Studentinnen signifikant mehr Angstsymptome an als westdeutsche. Daneben gab es Hinweise auf einen stärker ausgeprägten Schlankheitswunsch der Ostdeutschen. Insgesamt wiesen die ostdeutschen Studentinnen in allen Verfahren höhere Mittelwerte auf als 10 Jahre vorher. Diskussion: Die Studie bestätigt vorliegende Ergebnisse, wonach nur geringe Unterschiede in der psychischen Befindlichkeit in Ost- und Westdeutschland bestehen. Allerdings sind in den letzten 10 Jahren Veränderungen geschehen, die aufgrund fehlender Längsschnittstudien nicht eruiert wurden. Die höhere Ausprägung der Angstsymptomatik der ostdeutschen Studentinnen wird als ein Sozialisationsergebnis interpretiert.

\section{Key Words}

East-West-comparison · Psychological symptoms Mood state $\cdot$ University students

\section{Summary}

Psychological Symptoms in Female University Students in East and West Germany: A Replication after 10 Years

Background: After the German unification, several studies have focused on differences in the psychological health of people in East and West Germany. The current study is a replication of the Basten et al. study [Psychological wellbeing and health in a unified Germany: An East-West-comparison of two student samples. Verhaltenstherapie 1994;4:90-95] 10 years after the original research of 1991/1992. It investigated differences in depression, anxiety and bodily symptoms as well as problematic eating behaviour in female university students in Dresden (East Germany) and Marburg (West Germany). Subjects and Methods: From October 2001 to April 2002, we investigated psychological symptoms and eating behaviour in students of the universities of Dresden and Marburg. The current report includes data of 221 female students. We used the same standardized measures as had been used in the original research. Results: In contrast to the original study, students from West Germany did no longer exhibit higher depression scores. Instead, students from East Germany showed significantly increased anxiety symptoms. Furthermore, there was a trend towards a higher drive for thinness in East German students. In all, students from Dresden showed higher mean values in all measures than 10 years before. Discussion: This study corroborates results that there are only minor differences in psychological health between people in East and West Germany. However, our findings point out that during the past decade subtle changes might have occurred which have not been detected through lack of longitudinal investigations. The higher level of anxiety in East German students could be a result of socialisation.

\begin{tabular}{ll}
\hline KARGER & ○ 2003 S. Karger GmbH, Freiburg \\
Fax +49761 4520714 & Accessible online at: \\
$\begin{array}{l}\text { E-mail Information@Karger.de } \\
\text { www.karger.com }\end{array}$ & www.karger.com/ver \\
&
\end{tabular}

Dipl.-Psych. Andreas Dinkel

Universitätsklinikum Carl Gustav Carus an der TU Dresden Medizinische Psychologie

Fetscherstr. 74, D-01307 Dresden

Tel. +49 351 458-4029, Fax -5526

E-mail andreas.dinkel@mailbox.tu-dresden.de; www.medpsy.de 


\section{Einleitung}

Der Fall der Berliner Mauer und die Wiedervereinigung der beiden jahrzehntelang geteilten deutschen Staaten lösten eine Phase sozialen Wandels aus, die bis heute andauert [vgl. Héon-Klin et al., 2001; Hessel et al., 1999]. Die Auswirkungen der Wiedervereinigung haben auch die psychologische Forschung beschäftigt [vgl. Berth, 2001; www.wiedervereinigung.de], unter anderem bezüglich der Folgen für die physische und psychische Gesundheit der Deutschen. Anfang der 1990er Jahre setzte in den neuen Bundesländern eine Phase ein, die Nolte und McKee [2000, S. 1094] als 'a period of sustained improvement in health that exceeded even the most optimistic predictions' charakterisieren. Nach Nolte und McKee sind vor allem die veränderte Verfügbarkeit von Nahrungsmitteln und eine verbesserte medizinische Versorgung für eine bessere körperliche Gesundheit der Menschen in der ehemaligen DDR verantwortlich. Gleichzeitig weisen sie aber darauf hin, dass das Gesundheitssystem der ehemaligen DDR auch Vorteile hatte, die nun verloren zu gehen scheinen, was sich z.B. in gesunkenen Impfraten bei Kindern zeigt. Allerdings besteht noch immer eine höhere Rate an körperlichen Erkrankungen in Ostdeutschland, die zum Teil auch Ausdruck einer unterschiedlichen Versorgungsdichte ist [Brenner, 2001]. Daneben führte die Wiedervereinigung bei vielen Menschen in den neuen Bundesländern zu Verunsicherungen und persönlichen Krisen. Der Verlust des Arbeitsplatzes stellt bis heute wohl die gravierendste dieser Krisen dar. Die Umgestaltung der persönlichen Lebensumwelt - ob positiv oder negativ erlebt - konnte kaum ohne Folgen für die psychische Befindlichkeit bleiben. Auch wenn es sicherlich Personen gibt, für die die Wende in der ehemaligen DDR zu einer persönlichen Lebenssituation führte, die mit beeinträchtigtem psychischen Befinden verbunden war [vgl. Arenz, 1995], zeigen größer angelegte Untersuchungen in der Regel - zum Erstaunen der meisten Autoren - keine negativen Auswirkungen der Wiedervereinigung auf die seelische Gesundheit der Ostdeutschen und keine größere psychische Belastung in Ostdeutschland als in Westdeutschland. So konnten Achberger et al. [1999] im Jahr nach dem Fall der Mauer keine unterschiedliche Prävalenz psychischer Störungen bei ost- und westdeutschen Patienten der Primärversorgung feststellen. Priebe und Bröker [2000] fanden keine negativen Veränderungen bei ostdeutschen Schizophreniepatienten in den ersten 5 Jahren nach dem Fall der Berliner Mauer. Frommer [2002] berichtet über eine Studie an Patienten psychosomatischer Universitätsabteilungen in Düsseldorf und Magdeburg. Hier zeigte sich kein signifikanter Unterschied in der generellen Symptombelastung in der Symptomcheckliste von Derogatis (SCL-90-R) [Franke, 1995], wenngleich westdeutsche Patienten eher depressive Symptome angaben und ostdeutsche Patienten eher Angstsymptome.

Auch Studien in der Allgemeinbevölkerung zeigten keine deutlich negativere psychische Verfassung der Menschen in
Ostdeutschland. Wittchen et al. [1994] fanden in einer Reanalyse von vier Untersuchungen aus den Jahren 1990-1992 kaum Anhaltspunkte für ein schlechteres psychisches Befinden von Jugendlichen und Erwachsenen in Ostdeutschland. Die deutlichsten Hinweise für eine höhere Prävalenz in Ostdeutschland ergaben sich für einzelne depressionstypische und psychovegetative Symptome in der Jugendlichenstichprobe. Weiterhin zeigten zwei bevölkerungsrepräsentative Erhebungen im Jahr 1996 [Hessel et al., 2001; Jakobsen et al., 1999] keine wesentlichen Unterschiede in der psychischen Befindlichkeit zwischen Ost- und Westdeutschland. In einer Längsschnittstudie bei älteren Menschen in Heidelberg und Leipzig zeigte sich weder zum ersten Erhebungszeitpunkt (Anfang der 1990er Jahre) noch zum zweiten Erhebungszeitpunkt (Ende der 1990er Jahre) ein signifikanter Unterschied in der Depressivität der Studienteilnehmer [Ettrich et al., 1999]. Zwei Studien aus den mittleren bis späten 1990er Jahren erbrachten sogar, dass die psychische Befindlichkeit in Ostdeutschland besser ist als in Westdeutschland. Kruse et al. [2001] fanden bei jungen Erwachsenen in Rostock eine bessere seelische Gesundheit als bei jungen Erwachsenen in Mannheim. In einem bevölkerungsrepräsentativen Survey ermittelten Wittchen et al. [1999] eine höhere Prävalenz von affektiven und somatoformen Störungen in Westdeutschland und keine Prävalenzunterschiede zwischen Ost- und Westdeutschland bei Angststörungen nach DSM-IV [Saß et al., 1996]. Lediglich in Bezug auf Angstsymptome zeigen die Untersuchungen von Hänsgen et al. [1992] aus dem Jahr 1990 und Margraf und Poldrack [2000] aus dem Jahr 1994 eine höhere Ausprägung bei Ostdeutschen. Ebenso berichten Brähler et al. [2002] über höhere Angstwerte Ostdeutscher in der Hospital Anxiety and Depression Scale (dt. Version: HADS-D), die sie in einer 1998 durchgeführten Repräsentativerhebung ermittelten [vgl. Hinz und Schwarz, 2001].

Eine weitere Untersuchung zum psychischen Befinden in Ostund Westdeutschland stammt von Basten et al. [1994] und wurde Ende 1991/Anfang 1992 durchgeführt. Diese Untersuchung ist insofern herauszuheben, als sie speziell bei Studentinnen durchgeführt wurde. Zudem wurde hier auch das Essverhalten thematisiert. Die Autoren erhoben das Ausmaß gezügelten Essverhaltens und verschiedene psychologische Merkmale, die in Zusammenhang mit gestörtem Essverhalten stehen. Die Ergebnisse der Untersuchung, die an 89 Dresdner und 178 Marburger Studentinnen durchgeführt wurde, erbrachten signifikant höhere Depressivitäts- und Somatisierungswerte bei den Marburger Studentinnen. Die Marburger Studentinnen zeigten außerdem ein tendenziell höheres Aus$\mathrm{ma} ß$ an gezügeltem Essverhalten. Weiterhin waren die Mittelwerte in allen eingesetzten standardisierten Verfahren auf Seiten der Marburger Studentinnen höher. Während die Autoren von der höheren Depressivität und Somatisierung der westdeutschen Stichprobe überrascht waren, entsprachen die auffälligeren Werte beim gestörten Essverhalten ihren Erwartungen. Basten et al. vermuteten, dass sich - da die ostdeutschen 
Tab. 1. Mittelwerte und Standardabweichungen der Untersuchungsverfahren in Abhängigkeit des Untersuchungsortes 1991/1992 [Basten et al., 1994] und 2001/2002; Mittelwertvergleich für die beiden Erhebungszeitpunkte

\begin{tabular}{|c|c|c|c|c|c|c|c|c|c|c|}
\hline & \multicolumn{2}{|c|}{$\begin{array}{l}\text { Dresden }(1991 / 1992) \\
\mathrm{n}=89\end{array}$} & \multicolumn{2}{|c|}{$\begin{array}{l}\text { Marburg }(1991 / 1992) \\
\mathrm{n}=178\end{array}$} & \multicolumn{2}{|c|}{$\begin{array}{l}\text { Dresden }(2001 / 2002) \\
\mathrm{n}=140-145\end{array}$} & \multicolumn{2}{|c|}{$\begin{array}{l}\text { Marburg }(2001 / 2002) \\
\mathrm{n}=73-76\end{array}$} & Dresden & Marburg \\
\hline & M & SD & M & SD & M & SD & M & SD & $\mathrm{t}-\mathrm{V}$ & \\
\hline ADS & 11,1 & 7,6 & 15,1 & 9,5 & 14,7 & 9,5 & 13,5 & 9,0 & $3,03^{* *}$ & $-1,25$ \\
\hline BAI & 6,0 & 6,1 & 7,4 & 6,6 & 11,2 & 8,6 & 7,9 & 6,2 & $5,00^{* *}$ & 0,56 \\
\hline SCL & 15,3 & 3,4 & 16,9 & 3,9 & 6,9 & 5,9 & 5,1 & 4,0 & $-12,24^{* * * *}$ & $-21,81^{* * * *}$ \\
\hline RS & 8,6 & 4,5 & 10,0 & 5,1 & 12,3 & 6,2 & 11,1 & 5,5 & $4,89^{* *}$ & 1,54 \\
\hline EDI $^{\mathrm{a}}$ & 9,2 & 10,2 & 10,3 & 10,7 & 21,0 & 7,7 & 18,9 & 6,4 & $9,80^{* * * *}$ & $6,41^{* *}$ \\
\hline
\end{tabular}

${ }^{a}$ Mittelwert über die Skalen DT, B, BD.

${ }^{\mathrm{b}}$ Die signifikanten Unterschiede sind auch nach Bonferroni-Korrektur (notwendiges Signifikanzniveau von $\alpha=0,01$ ) gültig.

${ }^{* *} \mathrm{p}<0,01 ;{ }^{* * *} \mathrm{p}<0,001$.

Frauen noch nicht lange einem Überangebot von Nahrungsmitteln sowie der Propagierung eines Schlankheitsideals ausgesetzt waren - erst zukünftig das Ausmaß gestörten Essverhaltens in Ostdeutschland dem in Westdeutschland anpassen werde.

Das Ziel der vorliegenden Untersuchung ist die Replikation der Originalstudie von Basten et al. [1994] 10 Jahre später. Es soll untersucht werden, inwieweit sich die psychische Befindlichkeit von Studentinnen in Dresden und Marburg unterscheidet. Von besonderem Interesse ist dabei, ob es Differenzen bei Variablen gibt, die als Indikatoren eines gestörten Essverhaltens angesehen werden können.

\section{Probanden und Methode}

\section{Durchführung der Studie und Stichprobenbeschreibung}

Zwischen Oktober 2001 und April 2002 wurde an den Universitäten Dresden und Marburg eine Untersuchung zu psychischen Symptomen und Essverhalten bei Studenten durchgeführt. Die Teilnehmer wurden zum einen durch Aushang gewonnen, zum anderen wurden die Fragebögen in Vorlesungen verteilt. Im Unterschied zum Vorgehen von Basten et al. [1994] wurde die Teilnahme nicht vergütet; Studierenden der Psychologie wurden bei Bedarf Versuchspersonenstunden bescheinigt.

Insgesamt nahmen 406 Studierende an der Untersuchung teil. Die männlichen Studenten wurden von der weiteren Datenanalyse ausgeschlossen, da in der Originalstudie von Basten et al. nur Studentinnen betrachtet wurden. Dies resultierte in unserer Studie in einem Sample von 301 Frauen. Davon waren 16 zum Studium aus den alten in die neuen Bundesländer gewechselt und 4 aus den neuen Bundesländern in die alten; 7 Personen machten hierzu keine Angabe. Diese 27 Personen wurden aus der Stichprobe ausgeschlossen. Analog zu Basten et al. wurden die Dresdner und die Marburger Stichprobe hinsichtlich des Anteils an Psychologiestudentinnen parallelisiert. Der resultierende Anteil betrug in Dresden $73,8 \%$, in Marburg 73,7\%. In die Analysen gingen somit 145 Dresdner und 76 Marburger Studentinnen ein. Das Durchschnittsalter der Dresdner Teilstichprobe betrug 21,6 Jahre (Standardabweichung SD = 3,5, Range 18-42), die Marburger Teilstichprobe war im Mittel 23,7 Jahre alt ( $\mathrm{SD}=4,6$, Range 18-43). Dieser Altersunterschied ist statistisch signifikant, $\mathrm{t}(221)=121,05, \mathrm{p}<0,01$. Ein Grund hierfür ist, dass sich die Semesterverteilung in den beiden Stichproben unterschied. Während sich 81,3\% der Dresdner Stichprobe im 1.-3. Semester befanden, waren es bei den Marburger Studentinnen nur 35,1\%.

\section{Erhebungsinstrumente}

Es kamen dieselben Verfahren zum Einsatz, die von Basten et al. genutzt wurden.

Allgemeine Depressions Skala (ADS): Die ADS [Hautzinger und Bailer, 1992] erfasst mittels 20 Items die Häufigkeit depressiver Symptome in der vergangenen Woche. Die interne Konsistenz bei Personen der Allgemeinbevölkerung wird mit 0,89 angegeben.

Beck Angst-Inventar (BAI): Das BAI [Margraf und Ehlers, in Vorbereitung] enthält 21 Items zu somatischen und kognitiven Angstsymptomen, deren Intensität für die vergangene Woche eingeschätzt wird. Borden et al. [1991] berichten ein Cronbachs alpha von 0,91 in einer nichtklinischen Studentenstichprobe.

SCL-90-R: Wir verwendeten die Somatisierungsskala der SCL-90-R [Franke, 1995], die körperliche Beschwerden während der letzten 7 Tage erfasst. Die interne Konsistenz der Skala in einer aktuellen bevölkerungsrepräsentativen Stichprobe beträgt 0,83 [Hessel et al., 2001].

Restraint Scale (RS): Die RS von Herman und Polivy [1980] erfasst mittels 10 Items das Ausmaß gezügelten Essverhaltens. Die interne Konsistenz der RS in unserer Gesamtstudentenstichprobe betrug 0,83 [Dinkel et al., im Druck].

Eating Disorder Inventory (EDI): Das EDI [Thiel und Paul, 1988] erfasst für Essstörungen relevante Einstellungen und Erlebensweisen. Wir setzten die 3 zentralen Skalen des EDI ein: drive for thinnes (DT), bulimia (B) und body dissatisfaction (BD) mit insgesamt 23 Items. Thiel und Paul geben für die 3 Skalen eine interne Konsistenz von $0,72-0,89$ bei Frauen ohne Hinweise auf eine Essstörung an.

\section{Ergebnisse}

Ein Vergleich der Mittelwerte und Standardabweichungen der Untersuchungsvariablen der Originaluntersuchung 1991/1992 und der vorliegenden Replikation zeigt, dass 10 Jahre später vor allem in der Dresdner Studentinnenstichprobe in allen Verfahren deutlich andere Mittelwerte vorliegen. Der t-Test für unabhängige Stichproben zeigt, dass diese Veränderungen bei allen Verfahren signifikant sind. Mit Ausnahme der Somatisierungsskala der SCL-90-R liegen die Mittelwerte höher als kurz nach der Wende. Für die Marburger Stichprobe fallen die 
Tab. 2. Interkorrelationen der Untersuchungsvariablen in der Gesamtstichprobe $(\mathrm{n}=212-221)$

\begin{tabular}{llllll}
\hline & ADS & BAI & SCL & RS & EDI \\
\hline ADS & - & 0,70 & 0,55 & 0,45 & 0,45 \\
BAI & & - & 0,79 & 0,43 & 0,43 \\
SCL & & & - & 0,36 & 0,39 \\
RS & & & & - & 0,85 \\
EDI $^{\text {a }}$ & & & & & - \\
\hline
\end{tabular}

askalen DT, B, BD.

Alle Interkorrelationen sind signifikant bei $\mathrm{p}<0,001$.

Unterschiede zwischen den beiden Studien geringer aus. Hier ist ebenso ein im Vergleich zu 10 Jahren zuvor signifikant niedrigerer Mittelwert in der Somatisierungsskala festzustellen. Daneben ist ein signifikant höherer Wert im EDI zu verzeichnen. Die Veränderungen in den anderen Verfahren sind nicht signifikant (vgl. Tab. 1).

Für die statistischen Analysen bzgl. des Ost-West-Vergleichs orientierten wir uns am Vorgehen von Basten et al. Entsprechend ihrer Studie fand sich auch in unserer Stichprobe ein signifikanter Altersunterschied, wobei wiederum die Dresdner Stichprobe jünger war (siehe oben). Dies legte nahe, ana$\log$ der Originaluntersuchung eine multivariate Kovarianzanalyse mit dem Untersuchungsort als unabhängiger Variable und dem Alter als Kovariate durchzuführen. Allerdings entschlossen wir uns, zunächst die bivariaten Korrelationen zwischen dem Alter und den abhängigen Variablen zu betrachten, da die Stichproben durch eine vergleichsweise enge Altersspanne gekennzeichnet waren und für einige der verwendeten Verfahren keine Alterseffekte berichtet werden (ADS, Hautzinger und Bailer [1992]; RS, Dinkel et al. [im Druck]). Dabei zeigte sich nur für den BAI eine signifikante Korrelation mit dem Alter $(r=-0,19, p<0,01)$, so dass wir darauf verzichteten, Alter als Kovariate in die Analysen aufzunehmen.

Bei der folgenden MANOVA wurden 9 Studentinnen wegen unzulässiger Missings in einem der Verfahren ausgeschlossen, so dass die Stichprobengrößen $n=139$ (Dresden) bzw. $n=73$ (Marburg) betrugen. Die Interkorrelationen der Untersuchungsvariablen sind in Tabelle 2 dargestellt.

Das Ergebnis der MANOVA zeigte, dass sich beide Stichproben signifikant voneinander unterscheiden, $F(5,206)=2,86$, $p$ $<0,05$. Die nachfolgende Betrachtung der univariaten FWerte erlaubt eine Aussage darüber, in welchem Ausmaß die einzelnen Variablen am Zustandekommen der Gesamtsignifikanz beteiligt sind. Hierbei ergab sich ein signifikanter Unterschied zwischen den Dresdner und Marburger Studentinnen für Angst (BAI; F $(1,210)=7,28, \mathrm{p}<0,01)$, Somatisierung $(\mathrm{SCL}-90-\mathrm{R} ; \mathrm{F}(1,210)=4,86, \mathrm{p}<0,05)$ und gestörtes Essverhalten $(\mathrm{EDI} ; \mathrm{F}(1,210)=4,16, \mathrm{p}<0,05)$. Aufgrund der moderaten bis hohen Interkorrelationen der abhängigen Variablen
Tab. 3. Mittelwerte und Standardabweichungen der drei Skalen des EDI in Abhängigkeit vom Untersuchungsort sowie Interkorrelationen der Variablen in der Gesamtstichprobe

\begin{tabular}{|c|c|c|c|c|c|c|c|}
\hline & \multicolumn{2}{|c|}{$\begin{array}{l}\text { Dresden } \\
(\mathrm{n}=142-143)\end{array}$} & \multicolumn{2}{|c|}{$\begin{array}{l}\text { Marburg } \\
(\mathrm{n}=72-75)\end{array}$} & \multirow[t]{2}{*}{ DT } & \multirow[t]{2}{*}{ B } & \multirow[t]{2}{*}{ BD } \\
\hline & M & SD & M & SD & & & \\
\hline DT & 19,0 & 8,8 & 16,2 & 7,4 & - & 0,63 & 0,74 \\
\hline B & 12,6 & 6,4 & 11,5 & 3,4 & & - & 0,52 \\
\hline $\mathrm{BD}$ & 31,9 & 11,2 & 29,0 & 10,9 & & & - \\
\hline
\end{tabular}

Alle Interkorrelationen sind signifikant bei $\mathrm{p}<0,001$.

führten wir, analog zu Basten et al., eine Roy-Bargmann Stepdown Analyse durch [vgl. Tabachnik und Fidell, 2001]. Diese prüft, welche Unterschiede erhalten bleiben, wenn die Skalen jeweils als Kovariate berücksichtigt werden. Es ergab sich lediglich ein signifikanter Stepdown F-Wert für den BAI (F (1, 209) $=10,29, \mathrm{p}<0,01)$.

\section{Zusätzliche Analysen}

Bei dem signifikanten Unterschied zwischen Dresdner und Marburger Studentinnen im BAI gilt es zu bedenken, dass gerade der BAI einen signifikanten Zusammenhang mit dem Alter aufweist, wie die zuvor durchgeführten bivariaten Korrelationsanalysen zeigten. Daher führten wir zusätzlich eine univariate Kovarianzanalyse für den BAI mit dem Untersuchungsort als unabhängiger Variable und dem Alter als Kovariate durch, wobei die Kovariate als erstes in die Analyse einging. Aufgrund unzulässiger Missings wurde eine Studentin (Marburg) aus der Analyse ausgeschlossen. Das Ergebnis der ANCOVA zeigte auch nach Kontrolle des Alters einen signifikanten Unterschied im BAI zwischen den beiden Stichproben $(\mathrm{F}(1,219)=5,22, \mathrm{p}<0,05)$.

Die Berechnung eines Mittelwertes für die drei hier berücksichtigten EDI-Skalen (DT, B, BD) ist ungewöhnlich. In der Regel werden die drei Skalen gesondert betrachtet. Deshalb untersuchten wir zusätzlich, inwieweit sich die Dresdner und Marburger Stichprobe in diesen drei Skalen voneinander unterscheiden. Die Mittelwerte, Streuungen und Interkorrelationen dieser drei Skalen sind in Tabelle 3 dargestellt.

Bei Betrachtung der bivariaten Korrelationen zwischen den drei Skalen und dem Alter der Studentinnen wurde deutlich, dass nur für die Skala DT eine signifikante Korrelation besteht $(\mathrm{r}=-0,15, \mathrm{p}<0,05)$. Da nur eine von drei Skalen schwach signifikant mit dem Alter zusammenhing, verzichteten wir darauf, Alter als Kovariate aufzunehmen. Aufgrund unzulässiger Missings betrugen die Stichprobengrößen $\mathrm{n}=138$ (Dresden) bzw. $\mathrm{n}=70$ (Marburg). Die MANOVA mit den Skalen DT, B und BD als abhängigen Variablen erbrachte kei- 
nen signifikanten Unterschied zwischen den Dresdner und Marburger Studentinnen $(\mathrm{F}(3,204)=1,68, \mathrm{~ns})$. Allerdings zeigte sich ein signifikanter univariater F-Wert für die Skala DT $(\mathrm{F}(1,206)=5,00, \mathrm{p}<0,05)$, der auch in der Roy-Bargmann Stepdown Analyse signifikant blieb $(\mathrm{p}<0,05)$.

Aufgrund dieses teilweise signifikanten Ergebnisses, das auf einen Unterschied im Schlankheitswunsch zwischen Dresdner und Marburger Studentinnen hinweist, und der Vermutung von Basten et al. [1994], dass die Dresdner Studentinnen eventuell erst in Zukunft ähnlich «erfolgreich» in der Kalorienreduktion sein würden wie die Marburger Studentinnen, untersuchten wir abschließend, ob sich beide Stichproben im Body Mass Index (BMI; Körpergewicht [kg] dividiert durch Körpergröße $[\mathrm{m}]^{2}$ ) unterschieden, den wir zusätzlich erhoben haben. Aufgrund fehlender Werte lag die Stichprobengröße bei $\mathrm{n}=144$ (Dresden) bzw. $\mathrm{n}=74$ (Marburg). Der mittlere BMI der Dresdner Stichprobe betrug 20,9 (SD = 2,0, Range 16,2-26,8), die Marburger Stichprobe wies einen mittleren BMI von 21,2 auf (SD = 2,9, Range 16,8-30,8). Der BMI korrelierte in der Gesamtstichprobe signifikant mit dem Alter ( $r$ $=0,19, \mathrm{p}<0,01)$. Aufgrund der niedrigen Korrelation verzichteten wir darauf, Alter als Kovariate aufzunehmen und führten stattdessen einen Mittelwertvergleich durch. Dieser erbrachte, dass sich beide Stichproben nicht signifikant im BMI unterscheiden $(\mathrm{t}(218)=-0,91, \mathrm{~ns})$.

\section{Diskussion}

Die vorliegende Studie untersuchte Unterschiede in der psychischen Befindlichkeit bei Studentinnen in Ost- und Westdeutschland 10 Jahre nach der Originaluntersuchung von Basten et al. [1994]. Dabei zeigten sich 2001/2002 deutlich andere Ergebnisse als kurz nach der deutschen Wiedervereinigung.

So konnte die höhere Symptombelastung bei westdeutschen Studentinnen, wie sie 1991/1992 zu beobachten war, in der vorliegenden Studie nicht repliziert werden. Dies hängt vor allem mit einer im Vergleich zu 1991/1992 höheren Symptombelastung der Studentinnen in Ostdeutschland zusammen. Insofern haben sich die Verhältnisse genau umgekehrt. Wiesen 1991/1992 in allen Verfahren die westdeutschen Studentinnen höhere Mittelwerte auf, so geben 2001/2002 die ostdeutschen Studentinnen in allen Verfahren eine höhere Symptombelastung an. Dagegen hat sich die Symptombelastung westdeutscher Studentinnen wenig geändert. Die bei der Marburger Stichprobe im Vergleich zu 1991/1992 vorgefundenen Veränderungen in den Mittelwerten sind analog auch bei den Dresdner Studentinnen zu beobachten. In beiden Stichproben liegt die Beeinträchtigung durch primär somatische Symptome niedriger als vor 10 Jahren, während die Beeinträchtigung durch gewichts- und körperbezogene Probleme höher liegt. Die in diesen beiden Variablen, SCL und EDI, aufgetretenen Veränderungen sind von bedeutendem Ausmaß. Zum Teil kam es zu einer Mittelwertverschiebung von mehr als zwei
Standardabweichungen gegenüber 1991/1992 (s. SCL-Somatisierungsskala). Dieser Befund kann von uns nicht schlüssig interpretiert werden. Ein Methodenartefakt im Sinne fehlerhafter Kodier- und Berechnungsvorschriften schließen wir bei unseren Daten aus.

Im Unterschied zur Originalstudie fanden wir eine signifikant höhere Belastung durch Angstsymptome bei ostdeutschen Studentinnen. Dieses Ergebnis deckt sich mit der Arbeit von Margraf und Poldrack [2000], die ebenfalls den BAI einsetzten und in einer bevölkerungsrepräsentativen Untersuchung ein höheres Angstniveau bei der ostdeutschen Bevölkerung nachweisen konnten. Nichtsdestotrotz erscheint dieser Unterschied zwischen unseren beiden Studentinnenstichproben, der als einziger eindeutig statistische Signifikanz erreichte, erklärungsbedürftig. Eine Möglichkeit, die es zu bedenken gilt, liegt in den Merkmalen des eingesetzten Instrumentes. So weisen Cox et al. [1996] darauf hin, dass der BAI vor allem Paniksymptome erfasst und weniger Angst «im Allgemeinen». Eine geringe Sensitivität des BAI für Angststörungen berichten auch Hoyer et al. [2002]. Viele Items des BAI beziehen sich auf körpernahe Symptome, unter denen die ostdeutschen Studentinnen möglicherweise in höherem Maß leiden bzw. über die sie eher bereit sind, Auskunft zu erteilen. Wie Brähler et al. [2000] bei einer bevölkerungsrepräsentativen Untersuchung nachweisen konnten, geben Menschen in Ostdeutschland mehr körperliche Beschwerden an als Westdeutsche [vgl. auch Brähler et al., 2002]. Gegen diese Interpretation spricht jedoch, dass sich die Dresdner und Marburger Studentinnenstichproben unserer Untersuchung nicht in der Somatisierungsskala der SCL-90-R unterschieden, sobald die anderen Variablen kontrolliert wurden. In der Stepdown Analyse, in der nacheinander univariate Varianzanalysen unter wechselseitiger Kontrolle der weiteren Variablen durchgeführt werden, ergab sich keine statistische Signifikanz für die Somatisierungsskala. Dieses Ergebnismuster bestärkt die Annahme eines höheren generellen Angstniveaus bei ostdeutschen Studentinnen.

Margraf und Poldrack [2000] vermuten, dass das höhere Angstniveau in Ostdeutschland Ausdruck der Unkontrollierbarkeit und Unvorhersehbarkeit wesentlicher Lebensbereiche ist. Dabei kommt es weniger auf die tatsächliche, als auf die subjektiv erlebte Kontrolle und Vorhersehbarkeit an. Die Sozialisation im östlichen Teil Deutschlands kann dazu geführt haben, dass die Dresdner Studentinnen die Umwelt subjektiv als wechselhafter, bedrohlicher und somit unkontrollierbarer wahrnehmen als Studentinnen in Westdeutschland, auch wenn sie einen hohen Bildungsstand und potentiell gute Berufschancen haben - der Lebensbereich, der in Ostdeutschland sicherlich seit vielen Jahren als sehr problematisch und unvorhersehbar erlebt wird. Die meisten ostdeutschen Studentinnen unserer Stichprobe waren zum Zeitpunkt des Mauerfalls Kinder bzw. Jugendliche. Viele von ihnen werden die Anstrengungen ihrer Eltern bei der Bewältigung neuer Anforderungen mitbekommen haben. Beispielsweise fanden Born und 
Fuhrer [2000], dass Ostdeutsche in den Jahren nach der Wende mehr Veränderungen erlebten als Westdeutsche. Diese Veränderungen betrafen wichtige Bestimmungsstücke der persönlichen Identität, die es somit neu aufzubauen galt [vgl. Frommer, 2002]. Diese bedrohlichen Erfahrungen können sich auch in der Erziehung widergespiegelt haben. Wie Wolfradt et al. [1996] in einer Studie aus den Jahren 1994/1995 zeigen konnten, wiesen ostdeutsche Jugendliche, insbesondere Mädchen, im Alter von 13-17 Jahren höhere Angstwerte auf als westdeutsche. Bei dem in dieser Untersuchung eingesetzten Verfahren handelte es sich nicht um ein klinisches, sondern um ein persönlichkeitspsychologisches Angstmaß. Da auch Hänsgen et al. [1992] in ihrer Studie aus dem Jahr 1990 in einem persönlichkeitspsychologischen Verfahren höhere Angstwerte bei ostdeutschen Erwachsenen feststellen konnten, lässt sich die Hypothese aufstellen, dass das höhere Angstniveau der ostdeutschen Studentinnen auf einen langfristigen Sozialisationseffekt zurückzuführen ist.

Brähler et al. [2002] bemerken in diesem Zusammenhang, dass die Ergebnisse des Zusatzsurveys «Psychische Störungen» zum Bundesgesundheitssurvey [Wittchen et al., 1999] entgegengesetzte Ergebnisse liefern zu verschiedenen Studien - darunter auch der vorliegenden -, die eine höhere Angstsymptomatik in der ostdeutschen Bevölkerung fanden. Dies könnte ihrer Ansicht nach auf die Methode der Datengewinnung zurückzuführen sein. Während alle Studien, die höhere Angstwerte in Ostdeutschland fanden, Selbsteinschätzungsverfahren nutzten, kam im Zusatzsurvey «Psychische Störungen» ein computerunterstütztes klinisches Interview zum Einsatz. Brähler et al. [2002] geben zu bedenken, dass die divergenten Ergebnisse mit einem verminderten Mitteilungsbedürfnis oder geringerer Offenheit ostdeutscher Personen bei Interviews zusammenhängen könnten. Andererseits basiert unseres Erachtens die Diagnose einer psychischen Störung nicht allein auf den geäußerten Beschwerden, sondern auch auf dem erlebten Leidensdruck und den wahrgenommenen psychosozialen Einschränkungen. Es wäre möglich, dass eine dispositionell vorhandene höhere Ängstlichkeit der Ostdeutschen, wie dies einige Studien nahe legen [Hänsgen et al., 1992; Wolfradt et al., 1996], mit einem geringeren subjektiven Leidensdruck und geringeren Einschränkungen einhergeht, da Ostdeutsche habituell mehr Symptome erleben und somit auch dauerhaft damit umgehen müssen.

Bestätigen konnten wir in unserer Studie die Vermutung von Basten et al., wonach ostdeutsche Frauen erst zukünftig ein vergleichbares Maß an problematischen Verhaltensweisen im Zusammenhang mit Gewicht und Nahrungsaufnahme zeigen sollten. Die Werte in der RS und im EDI sind in der Dresdner Stichprobe im Vergleich zu 1991/1992 signifikant höher. Aktuell unterscheiden sich beide Stichproben jedoch kaum voneinander. Lediglich der signifikante univariate F-Wert in der EDI-Subskala DT legt nahe, dass ostdeutsche Studentinnen einen im Vergleich zu westdeutschen höheren Schlankeitswunsch haben. Dabei zeigen die ostdeutschen Studentinnen aber kein ausgeprägteres gezügeltes Essverhalten. Ebensowenig bestehen statistisch signifikante Unterschiede im BMI zwischen den Stichproben. Diese Ergebnisse in den Verfahren zum Essverhalten lassen vermuten, dass der von Basten et al. vorhergesagte Anpassungsprozess an westliche «Standards» im Sinne eines einvernehmlichen Schlankheitsideals tatsächlich stattgefunden hat, wenngleich unsere Studie keine direkte Aussage hierzu erlaubt.

Auch wenn die vorliegenden Querschnittuntersuchungen in der Regel keine deutlichen Belege für ein unterschiedliches Ausmaß an psychischer Beeinträchtigung in den alten und neuen Bundesländern liefern, so zeigen sie doch häufig nuancierte Differenzen. Aufgrund der Verschiedenheit der Ergebnisse dieser Untersuchungen lässt sich jedoch nur schwer beurteilen, welche dieser Differenzen als bedeutsam und systematisch einzuschätzen sind. Auch unsere Studie kann hierzu keine definitiven Aussagen machen. Allerdings unterstreichen unsere Ergebnisse die Notwendigkeit von Längsschnittuntersuchungen zu Unterschieden und Veränderungen der psychischen Befindlichkeit in Ost- und Westdeutschland. Mehr als 10 Jahre nach der Wiedervereinigung tritt nun die Frage in den Vordergrund, wie dieses historische Ereignis auf die nachrückende Generation wirkt. Angesichts eines Rückgangs der psychologischen Forschung zur Wiedervereinigung in den letzten Jahren [vgl. Berth, 2001] gilt es mit Nolte und McKee [2000, S. 1094] nichtsdestotrotz festzustellen: 'We need to know more about the impacts on health.'

\section{Literatur}

Achberger M, Linden M, Benkert O: Psychological distress and psychiatric disorders in primary health care patients in East and West Germany 1 year after the fall of the Berlin Wall. Soc Psychiatry Psychiatr Epidemiol 1999;34:195-201.

Arenz D: Wertewandel und Verunsicherung - einige Aspekte zur Wendeproblematik in den neuen Bundesländern. Verhaltenstherapie 1995;5:149-153.

Basten M, Florin I, Tuschen B, Wessels R, Hübner I, Bossong A, Schmidt S: Psychische Symptome und körperliche Gesundheit im vereinten Deutschland: Eine Ost-West-Vergleichsuntersuchung an einer studentischen Stichprobe. Verhaltenstherapie 1994;4:90-95.
Berth H: Psychologie und Wiedervereinigung. Eine quantitativ-deskriptive Analyse anhand der Veröffentlichungen. Psychol Beitr 2001;43:816-823.

Borden JW, Peterson DR, Jackson EA: The Beck Anxiety Inventory in nonclinical samples: Initial psychometric properties. J Psychopath Behav 1991;13:345356.

Born A, Fuhrer U: Identitätsstatus und Wohlbefinden in der Bewältigung gesellschaftlicher Transformation: Eine Studie in ost- und westdeutschen Zwillingsdörfern. Z Gesundheitspsychol 2000;8:39-48.
Brähler E, Schumacher J, Albani C, Strauß B: Wie bedeutsam sind Ost-West-Unterschiede? Eine Analyse von Ost-West-, Geschlechts- und Nord-Süd-Unterschieden bei psychologischen Testverfahren. Verhaltensther Psychosoz Prax 2002;34:301-312. Brähler E, Schumacher J, Brähler C: Erste gesamtdeutsche Normierung der Kurzform des Gießener Beschwerdebogens GBB-24. Psychother Psychol Med 2000;50:14-21.

Brenner G: Die «Gesundheitsmauer» besteht weiter. Versorgungsungleichgewicht in Ostdeutschland durch höhere Morbidität und niedrigeren Ressourceneinsatz Dtsch Arztebl 2001;98:A590-A593. 
Cox BJ, Cohen E, Direnfeld DM, Swinson RP: Does the Beck Anxiety Inventory measure anything beyond panic attack symptoms? Behav Res Ther 1996;34:949 954.

Dinkel A, Berth H, Exner C, Rief W, Balck F: Deutsche Adaptation der Restraint Scale zur Erfassung gezügelten Essverhaltens. Diagnostica, im Druck.

Ettrich KU, Huth M, Fischer-Cyrulies, A: Veränderungen von Einstellungs- und Persönlichkeitsmerkmalen im höheren Lebensalter und Beziehungen zum Gesundheitszustand im Ost-West-Verlgeich - Ergebnisse der ILSE-Studie; in Berth H, Brähler E (Hrsg.): Deutsch-deutsche Vergleiche. Psychologische Untersuchungen 10 Jahre nach dem Mauerfall. Berlin, Verlag für Wissenschaft und Forschung, 1999, pp 70-94.

Franke GH: Die Symptom-Checkliste von Derogatis Deutsche Version. Göttingen, Beltz, 1995.

Frommer J: Psychische Störungen durch globale gesellschaftliche Veränderungen. Zur politischen Traumatisierung der Bevölkerung in den neuen Bundesländern. Fortschr Neurol Psychiatr 2002;70:418-428.

Hänsgen KD, Kasielke E, Schmidt LR, Schwenkmezger P: Ostdeutsche und Westdeutsche im Vergleich: Emotionalität und Objektive Persönlichkeitsvariablen Z Klin Psychol Psychopathol Psychother 1992;40:346363.

Hautziger M, Bailer M: Allgemeine Depressions Skala. Göttingen, Beltz, 1992.

Héon-Klin V, Sieber E, Huebner J, Fullilove MT: The influence of geopolitical change on the well-being of a population: The Berlin Wall. Am J Public Health 2001; 91:369-374.
Herman CP, Polivy J: Restrained eating; in Stunkard AJ (ed): Obesity. Philadelphia, Saunders, 1980, pp. 208-225.

Hessel A, Geyer M, Brähler E (Hrsg): Gewinne und Verluste sozialen Wandels. Globalisierung und deutsche Wiedervereinigung aus psychosozialer Sicht. Opladen, Westdeutscher Verlag, 1999.

Hessel A, Schumacher J, Geyer M, Brähler E: Symptom-Checkliste SCL-90-R: Testtheoretische Überprüfung und Normierung an einer bevölkerungsrepräsentativen Stichprobe. Diagnostica 2001;47:27-39.

Hinz A, Schwarz R: Angst und Depression in der Allgemeinbevölkerung. Eine Normierungsstudie zur Hospital Anxiety and Depression Scale. Psychother Psychol Med 2001;51:193-200.

Hoyer J, Becker ES, Neumer S, Soeder U, Margraf J: Screening for anxiety in an epidemiological sample: Predictive accuracy of questionnaires. J Anxiety Disord 2002;16:113-134.

Jakobsen T, Zimmermann T, Rudolf G: Die „Wiedervereinigung" und ihre Gesundheitsfolgen oder psychische und psychosomatische Befindlichkeit in Ost- und Westdeutschland; in Hessel A, Geyer M, Brähler E (Hrsg): Gewinne und Verluste sozialen Wandels. Globalisierung und deutsche Wiedervereinigung aus psychosozialer Sicht. Opladen, Westdeutscher Verlag, 1999, pp. 200-211.

Kruse J, Meyer-Probst B, Reis O, Esser G, Ihle W: Seelische Gesundheit junger Erwachsener in Mannheim und Rostock. Z Klin Psychol Psychother 2001;30:55-
Margraf J, Ehlers A: Das Beck Angst-Inventar. Bern, Huber, in Vorbereitung.

Margraf J, Poldrack A: Angstsyndrome in Ost- und Westdeutschland: Eine repräsentative Bevölkerungserhebung. Z Klin Psychol Psychother 2000;29:157-169. Nolte E, McKee M: Ten years of German unification. We need to know more about the impacts on health. BMJ 2000;321:1094-1095.

Priebe S, Bröker M: Political change and course of schizophrenia in East Germany, 1984-1994. Soc Psychiatry Psychiatr Epidemiol 2000;35:255-258.

Saß H, Wittchen HU, Zaudig M: Diagnostisches und Statistisches Manual psychischer Störungen DSM-IV. Göttingen: Hogrefe.

Tabachnik BG, Fidell LS: Using multivariate statistics, ed 4. Boston, Allyn and Bacon, 2001

Thiel A, Paul T: Entwicklung einer deutschsprachigen Version des Eating-Disorder-Inventory (EDI). Z Diff Diagnost Psychol 1988;9:267-278.

Wittchen HU, Lachner G, Perkonigg A, Hoeltz J: Sind psychische Störungen in den neuen Bundesländern häufiger? Verhaltenstherapie 1994;4:96-103.

Wittchen HU, Müller N, Pfister H, Winter S, Schmidtkunz B: Affektive, somatoforme und Angststörungen in Deutschland - Erste Ergebnisse des bundesweiten Zusatzsurveys «Psychische Störungen». Gesundheitswesen 1999;61(S2):S216-S222.

Wolfradt U, Hellmeister G, Hansel B, Straube ER Ost-West-Unterschiede bei Jugendlichen hinsichtlich der Ausprägung verschiedener klinisch-psychologischer Persönlichkeitsmerkmale. Verhaltenstherapie 1996; 6:88-93. 